\title{
Study of the Young's Modulus in Microstructures through the Resonance Frequency Technique for Applications in Commercial CMOS Processes
}

\author{
Erick Araújo dos Santos ${ }^{a}$, Gustavo P. Rehder ${ }^{a}$ \\ ${ }^{a}$ Departamento de Engenharia de Sistemas Eletrônicos, Escola Politécnica da Universidade de São \\ Paulo, Avenida Prof. Luciano Gualberto - Travessa 3, 158, São Paulo, SP, Brazil
}

Received: April 30, 2017; Revised: September 19, 2017; Accepted: October 11, 2017

\begin{abstract}
The companies that manufacture devices with techniques inherited from microelectronics, called foundries, generally provide the electrical characteristics of the layers of their manufacturing processes, but they do not provide the mechanical parameters, which prevents the mechanical optimization of any design used in this manufacturing. Thus, the extraction of mechanical properties from the layers of a commercial process is important and it requires specific techniques and microstructures for this purpose. This work presents a study on the Young's modulus of cooper thin films using the resonance frequency technique to extract this parameter. The microstructures used for the application of the technique are cooper cantilevers with lengths of 100-700 $\mu \mathrm{m}$, width $40 \mu \mathrm{m}$ and thickness 2 $\mu \mathrm{m}$ suspended in such a way that it is possible to measure their resonant frequency. These structures are also simulated using the finite element method. The experimental results are compared with the simulations and presented equivalence.
\end{abstract}

Keywords: Young's Modulus, Mechanical Properties, Ressonance Frequency, Cooper Cantilevers, Commercial CMOS Processes, MEMS.

\section{Introduction}

Commercial Complementary Metal Oxide Semiconductor (CMOS) technology is basically a manufacturing process that uses microelectronic processes for the construction of devices that take into account design rules (maximum and minimum distances and dimensions) and specification of the technology (thickness of metallic layers and insulating layers) of each CMOS technology provided by the foundries, which are companies that implement this manufacturing technology at the commercial level. In this sense, the electrical characteristics of CMOS process materials and films, such as resistivity and dielectric constant, are usually provided by the foundries. However, its mechanical properties, such as Young's modulus, density and residual stress are not provided. The extraction of these parameters is not straightforward and often requires the use of different techniques to obtain them. One of these techniques is the resonant frequency measurement ${ }^{1}$. Its application consists of placing a cantilever in vibration and aligning a laser to its tip. An amplitude detector is placed close to this point to detect the laser light reflected by the cantilever tip so that changes in amplitude in the reflected light of the laser (due to changes in the vibrational amplitude of the cantilever) are picked up by the detector. Thus, using the Euler and Bernoulli model for clamped-free beams ${ }^{2}$, it is possible to relate the resonance frequency of the cantilever with its Young's modulus:

$$
f_{r}=\frac{\lambda_{i}^{2} t}{4 \pi l^{2}} \sqrt{\frac{E}{3 \rho}}
$$

where $f_{\mathrm{r}}$ is the resonant frequency of the cantilever, $\rho$, $E, l$ e $t$ are, respectively, the density, the Young's modulus, the length and the thickness of the cantilever and $\lambda_{i}$ is an eigenvalue, where the index $i$ is an integer which describes the number of the resonance mode; for the first mode, $\lambda_{i}=1.875$.

CMOS technology is used primarily for the manufacture of circuits/electronic devices, but it has been widely exploited for the development of Microelectromechanical Systems or $\mathrm{MEMS}^{3}$, as it is a mature and inexpensive technology and enables the direct integration of them with other external circuits, such as control, acquisition and treatment circuits of signals from MEMS. In this sense, MEMS use the layers of metallic films of this process as part of their electromechanical structure. The mechanical properties of a thin film are strongly determined by its deposition characteristics, such as the flux of gases used to form the film, temperature, among other parameters. Thus, knowing the mechanical properties of these layers becomes quite important for the optimization of the design of these devices.

In this study, in order to make this procedure feasible for a commercial manufacturing process, copper cantilevers were manufactured. Their resonant frequencies were obtained and compared with their values obtained analytically and through finite element simulation. 


\section{Materials and Research Method}

\subsection{Set up utilized}

The set up used to perform the resonant frequency measurements was mounted on an optical bench and is shown in Figure 1.

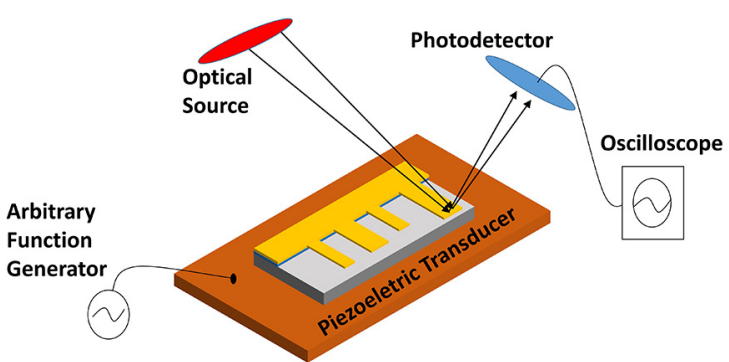

Figure 1. Set up used to measure the resonant frequencies of the cantilevers.

In order to vibrate the cantilevers, the samples were fixed to a piezoelectric transducer. This transducer is connected to a function generator, where square wave function is applied with frequencies between $25-50 \mathrm{~Hz}$, range that allows oscillation of the cantilevers and the visualization of their resonant frequencies in an oscilloscope. A laser source (optical fiber) is aligned to the cantilever tip, and a photodetector connected to the oscilloscope is placed close to this point to detect laser light reflected by the cantilever tip, so that the changes detected by the photodetector are displayed in function of frequency. Figure 2 shows the apparatus mounted and loaded with a sample of cantilevers.

A microscope was used to visualize the laser and the cantilevers, and the alignment between them was accomplished through the adjustment of a micropositioner. A GFC-8050 model function generator from GW and a DSO-X 2002A model oscilloscope from Agilent were used to excite the piezoelectric transducer and capture the signals from the laser, respectively. The photodetector (model L14G1) from Fairchild was used. From Figure 2 it is possible to visualize the acquired signal in the oscilloscope.

\subsection{Fabrication}

Copper cantilevers were manufactured in the Laboratory of Microelectronics of the Polytechnic School of University of São Paulo. Although the manufacture was not performed in a foundry, the specifications of CMOS technology of 0.35 $\mu \mathrm{m}$ of AMS foundry ${ }^{4}$ were considered in its stages, which makes possible its reproducibility in a commercial process. Groups of cantilevers with $40 \mu \mathrm{m}$ in width and lengths ranging from 100 to $700 \mu \mathrm{m}$ were manufactured. Obtaining the Young's modulus of cantilevers with different lengths reduces errors. Figure 3 shows the fabrication process of the cantilevers.

The manufacturing process of Figure 3 was started with the step (a), the sputtering deposition of $1 \mu \mathrm{m}$ of $\mathrm{SiO}_{2}$ on a Si wafer, followed by the process (b), the sputtering deposition of $\mathrm{Ti} / \mathrm{Cu}$ ( $\mathrm{Ti}$ is deposited only to improve $\mathrm{Cu}$ adhesion. The parameters of such depositions are shown in Table 1 and were chosen based on the Commercial CMOS process of AMS foundry ${ }^{4}$. After that, the step (c) was performed with the thickening of $\mathrm{Cu}$ by electrodeposition with applied electric current of 16,25 mA and electrodepostion time of 11 minutes and 34 seconds, followed by the step (d), the photolithography using AZ-1518 photoresist to pattern the cantilevers. In step (e), it was performed the etch of $\mathrm{Cu}$ to define the cantilevers and removal of the Ti layer in HF-H2O solution. Finally, steps (f) and (g) were performed, which

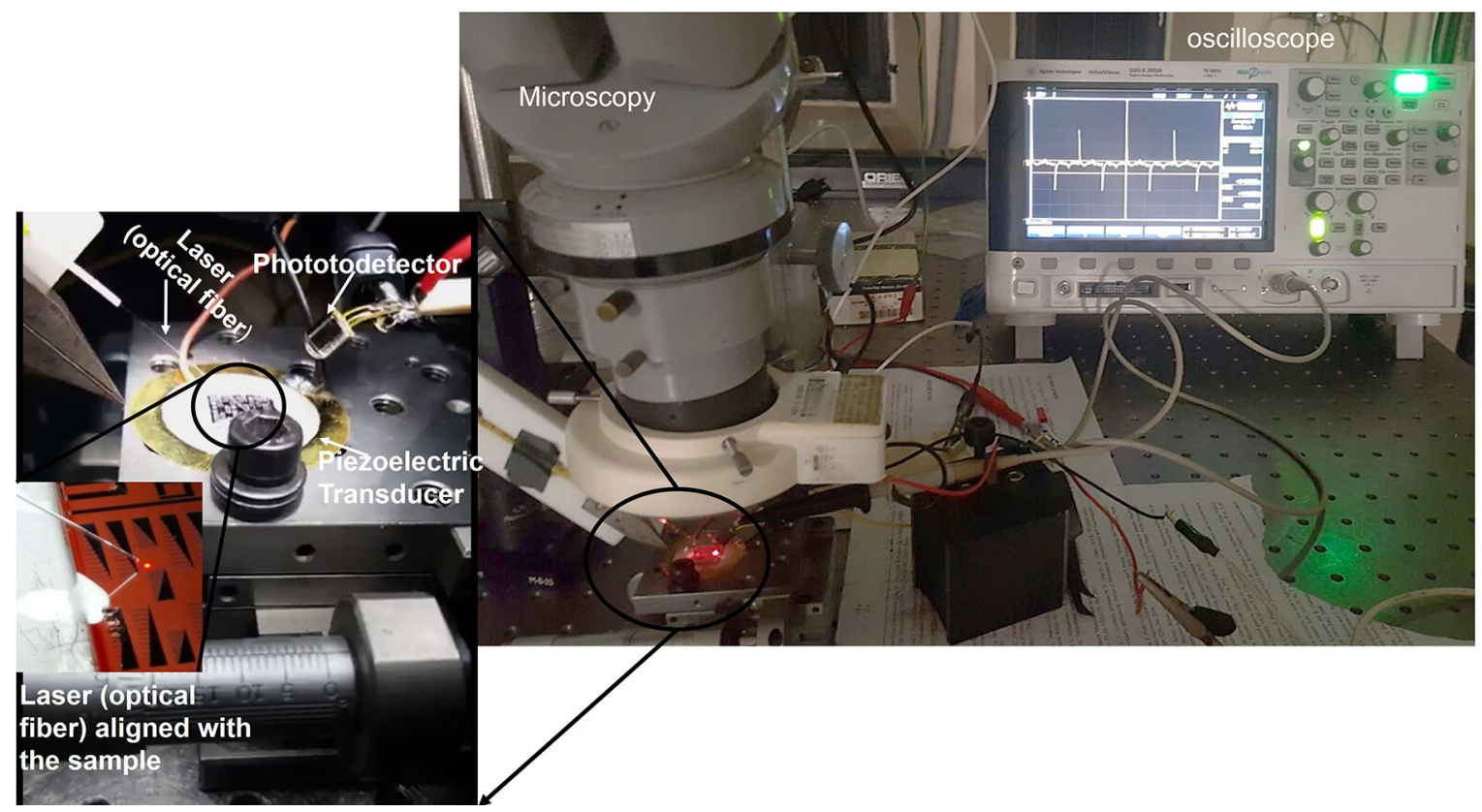

Figure 2. Apparatus for resonant frequency measurements, showing the equipment and components used. 


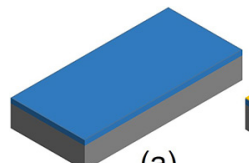

(a)

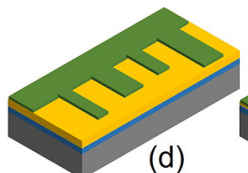

(d)

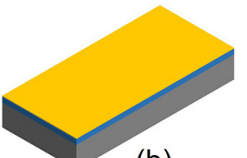

(b)

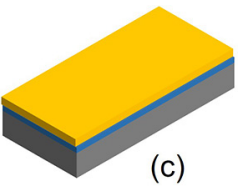

(c)

(e)

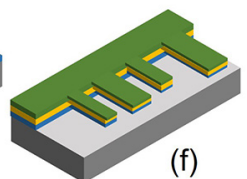

(f)
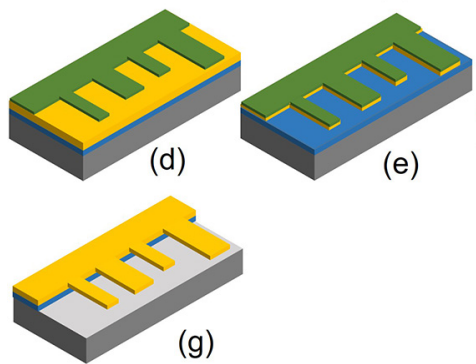

(g)

Figure 3. Fabrication process of the cantilevers.

Table 1. Parameters used in the sputtering deposition described in steps (a) and (b) of Figure 3.

\begin{tabular}{cccc}
\hline Parameter & $\mathrm{SiO}_{2}$ & $\mathrm{Ti}$ & $\mathrm{Cu}$ \\
\hline Argon flow (sccm) & 40 & 10 & 10 \\
Oxygen flow (sccm) & 60 & - & - \\
RF power (W) & 150 & 70 & 70 \\
Working pressure (mTorr) & 2 & 2,1 & 2,1 \\
Deposition time (minutes) & 60 & 5 & 5 \\
\hline
\end{tabular}

are the etch of the sacrificial $\mathrm{SiO} 2$ layer and the removal of the photoresist, respectively.

\section{Results and Discussion}

\subsection{Fabrication}

In Figure 4, it is possible to see the laser aligned with a cantilever. The detail in the lower left corner shows the cantilever view under the microscope. From this point the alignment of the laser with the cantilever tip was performed.

Figure 5 shows a micrograph of the groups of cantilevers with $40 \mu \mathrm{m}$ width.

The $\mathrm{SiO}_{2}$ layer, shown in letter $f$ of Figure 3, was used as a sacrificial layer. The etch step of this layer for release and suspension of copper cantilevers was the most critical to be carried out. Some cantilevers were broken to see if they were already suspended and without any remnants of the sacrificial layer of silicon oxide underneath. In some samples, the $\mathrm{SiO}_{2}$ layer adhered to the copper structures, which prevented their vibration. In Figure 6, a broken cantilever with the $\mathrm{SiO}_{2}$ film adhered to its structure is shown.

After the assurance that the structures were suspended, they were measured using the setup described above.

\subsection{Young's modulus}

Firstly, the copper density was obtained experimentally by its deposition on a square substrate of known size and weight. After this process, the weight of the electrodeposited

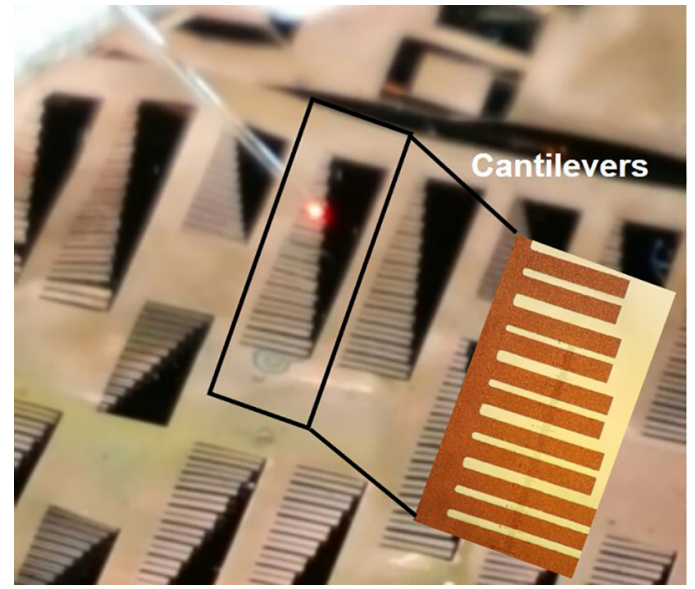

Figure 4. Laser aligned to a cantilever.

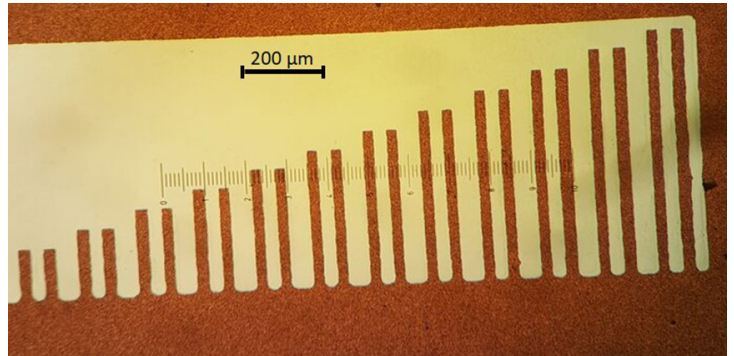

Figure 5. Groups of cantilevers with $40 \mu \mathrm{m}$ width and lengths between $100-700 \mu \mathrm{m}$.

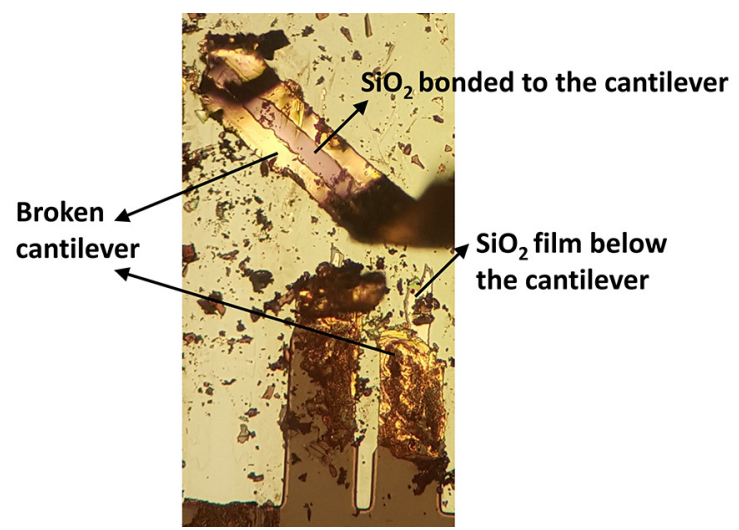

Figure 6. Broken cantilever with the $\mathrm{SiO}_{2}$ film adhered to its structure.

copper layer and its thickness were measured and a density $\rho=8409.5 \pm 225,5 \mathrm{~kg} / \mathrm{m}^{3}$ was found. So, the calculated and simulated resonance frequencies were obtained for different lengths of cantilevers. The data used for this were: Young's modulus $E=130 \mathrm{GPa}$ and thickness $t=2.19 \mu \mathrm{m}$. The Young's modulus was taken from the literature ${ }^{5}$. Copper thickness was measured with a profilometer. The simulation was performed with Ansys software using the finite element method. The cantilevers were simulated with the same dimensions used in manufacturing. 
Through Eq. (1), it can be observed that the resonance frequency is proportional to $1 / \mathrm{L}^{2}$. Therefore, by plotting the resonant frequency as a function of $1 / \mathrm{L}^{2}$ it is possible to obtain a straight line that can be used to extract the Young's modulus, reducing measurement error. Eq. (2) shows the relation of the Young's modulus in terms of the slope $m$.

$$
E=\frac{48 \pi^{2} \rho}{\lambda_{i}^{4} t^{2} m^{2}}
$$

In this way, the curves with calculated and simulated results of resonant frequencies were plotted, according to Figure 7, where the analytically calculated Young's modulus was $130 \mathrm{GPa}$, as expected, and the simulated modulus was $141.92 \mathrm{GPa}$.

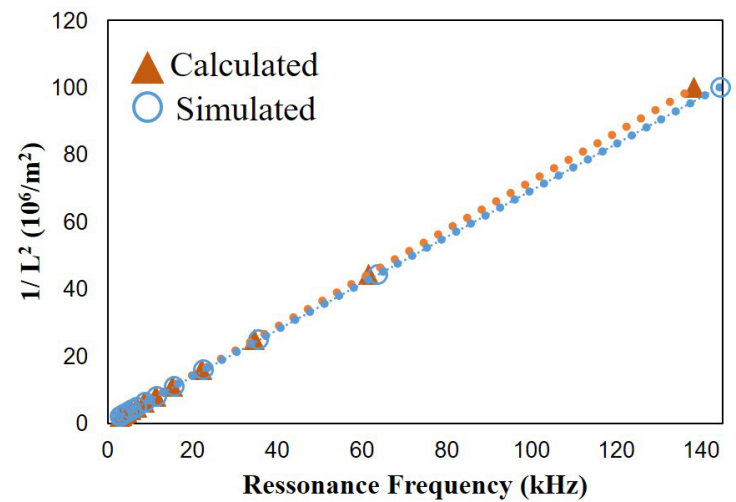

Figure 7. Curves with calculated and simulated results for the resonant frequency of copper cantilevers. Experimental measurement of the resonant frequency were performed on two groups of cantilevers. Figure 8 shows the two curves obtained with such groups of cantilevers. The Young's modulus obtained for group 1 was 125.35 $\mathrm{GPa}$ and for group 2 was $128.84 \mathrm{GPa}$, resulting in a final Young's modulus of $127.10 \pm 2.47 \mathrm{GPa}$.

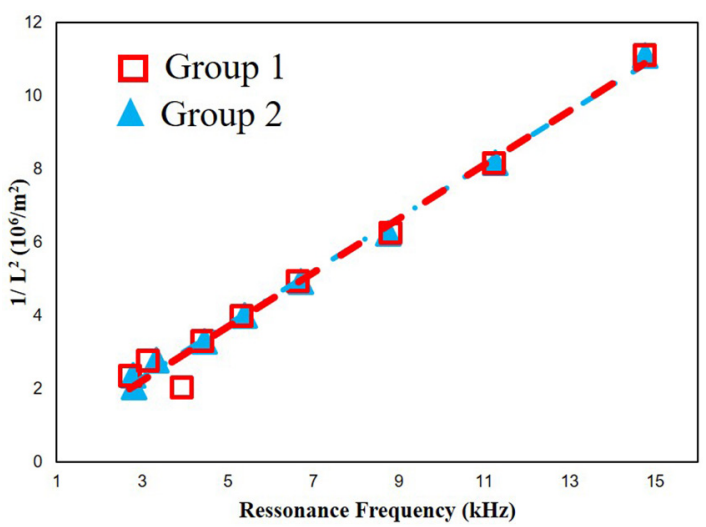

Figure 8. Resonant frequency of two groups of 40- $\mu \mathrm{m}$-wide cantilevers.

Different methods of obtaining the Young's modulus of copper are compared in Table 2. It is possible to see that the results presented here agree quite well with other literature results.
Table 2. Young's Modulus of copper obtained by different methods.

\begin{tabular}{ccc}
\hline Method & $\begin{array}{c}\text { Young's } \\
\text { modulus of } \mathrm{Cu} \\
(\mathrm{GPa})\end{array}$ & Reference \\
\hline Microindentation & $136.9 \pm 7.9$ & 6 \\
Nanoindentation & $125 \pm 5$ & 7 \\
Tensile and strain tests & 102.1 & 8 \\
Ultrasonic technique & 137.1 & 9 \\
Ressonant Frequency & $127.10 \pm 2.47$ & This work \\
\hline
\end{tabular}

\section{Conclusion}

It was possible to obtain the Young's modulus of the electrodeposited copper film through the resonant frequency analysis of cantilever beams. The cantilevers fabrication has been done successfully, however, special attention must be paid to the process of releasing these structures, since the control of the etching of the sacrificial layer is not an easy process. The experimental measurements were equivalent to the calculated and simulated results. Using the same measurement procedures adopted in this work, it is possible to obtain the Young's modulus of the metallic layers in a CMOS process.

\section{References}

1. Petersen KE, Guarnieri CR. Young's modulus measurements of thin films using micromechanics. Journal of Applied Physics. 1979;50(11):6761-6766.

2. Gere JM, Timoshenko SP. Mechanics of Materials. $4^{\text {th }}$ ed. Boston: PWS Publishing Company; 1997.

3. Bausells J, Carrabina J, Merlos A, Bota S, Samitier J. Mechanical sensors integrated in a comercial CMOS technology. Sensors and Actuators A: Physical. 1997;62(1-3):698-704.

4. AMS. Design Rules, Process Parameters, Assembly related and other Design Documents. Available from: http://asic.ams. com/cot/index.html. Access in: 13/3/2017.

5. MEMSNet. Material: Copper (Cu), bulk. Available from: $<\mathrm{https}$ ://www.memsnet.org/material/coppercubulk/>. Access in: $13 / 3 / 2017$.

6. Song X. Microstructure and mechanical properties of electrodeposited copper films. [Thesis]. Birmingham: University of Birmingham; 2011

7. Moharrami N, Oila A, Bull SJ. Extracting mechanical properties of copper coatings on oxided silicon substrates by nanoindentation. Journal of Physics D: Applied Physics. 2014;47(31):315104.

8. Tang Z, Pillay P, Omekanda AM, Li C, Cetinkaya C. Measurement of Young's modulus for switched reluctance motor vibration determination. In: Electric Machines and Drives Conference. IEEE International; 2003 Jun 1-4; Madison, WI, USA. p. 1590-1595.

9. Zhang S, Sakane M, Nagasawa T, Kobayashi K. Mechanical Properties of Copper Thin Films Used in Electronic Devices. Procedia Engineering. 2011;10:1497-1502. 